in vivo $31: 861-871(2017)$

doi:10.21873/invivo.11140

\title{
Comparing the Organs and Vasculature of the Head and Neck in Five Murine Species
}

\author{
MIN JAE KIM ${ }^{1 *}$, YOO YEON KIM ${ }^{2 *}$, JANET REN CHAO ${ }^{3}$, HAE SANG PARK ${ }^{1,4}$, JIWON CHANG ${ }^{1,4}$, \\ DAWOON OH${ }^{5}$, JAE JUN LEE ${ }^{4,6}$, TAE CHUN KANG ${ }^{7}$, JUN-GYO SUH $^{2}$ and JUN HO LEE ${ }^{1,4}$ \\ ${ }^{1}$ Department of Otorhinolaryngology-Head and Neck Surgery, College of Medicine, \\ Hallym University, Chuncheon, Republic of Korea; \\ ${ }^{2}$ Department of Medical Genetics, College of Medicine, Hallym University, Chuncheon, Republic of Korea; \\ ${ }^{3}$ School of Medicine, George Washington University, Washington, DC, U.S.A.; \\ ${ }^{4}$ Institute of New Frontier Research, Hallym University College of Medicine, Chuncheon, Republic of Korea; \\ ${ }^{5}$ Department of Anesthesiology and Pain Medicine, Dongtan Sacred Heart Hospital, \\ Hallym University, Dongtan, Republic of Korea; \\ ${ }^{6}$ Department of Anesthesiology and Pain Medicine, College of Medicine, \\ Hallym University, Chuncheon, Republic of Korea; \\ ${ }^{7}$ Department of Anatomy and Neurobiology, College of Medicine, Hallym University, Chuncheon, Republic of Korea
}

\begin{abstract}
Background/Aim: The purpose of the present study was to delineate the cervical and facial vascular and associated anatomy in five murine species, and compare them for optimal use in research studies focused on understanding the pathology and treatment of diseases in humans. Materials and Methods: The specific adult male animals examined were mice (C57BL/6J), rats (F344), mongolian gerbils (Merionesunguiculatus), hamsters (Syrian), and guinea pigs (Hartley). To stain the vasculature and organs, of the face and neck, each animal was systemically perfused using the vital stain, Trypan Blue. Following this step, the detailed anatomy of the head and neck could be easily visualized in all species. Results:
\end{abstract}

This article is freely accessible online.

*These Authors contributed equally to this study.

Correspondence to: Dr. Jun-Gyo Suh, Department of Medical Genetics, College of Medicine, Hallym University, 1, Hallymdaehakgil, Chuncheon, Gangwon-do, 24252, Republic of Korea. Tel: +82 332482692, Fax: +82 332482696, e-mail: jgsuh@hallym.ac.kr and Jun Ho Lee, Department of Otorhinolaryngology-Head and Neck Surgery, Chuncheon Sacred Heart Hospital, Hallym University College of Medicine, 77, Sakju-ro, Chuncheon, Gangwon-do, 200704, Republic of Korea. Tel: +82 332405181, Fax: +82 332412909, e-mail: zoonox@nate.com

Key Words: Comparative anatomy, blood vessels, rodentia, neck dissection, neck anatomy.
Unique morphological characteristics were demonstrated by comparing the five species, including symmetry of the common carotid origin bilaterally in the Mongolian Gerbil, a large submandibular gland in the hamster and an enlarged buccal branch in the Guinea Pig. In reviewing the anatomical details, this staining technique proves superior for direct surgical visualization and identification. Conclusion: The anatomical details provided through these five species atlas will help experimental researchers in the future to select the most appropriate animal model for specific laboratory studies aimed to improve our understanding and treatment of diseases in patients.

The murine species are often used as an in vivo model due to their relatively short developmental period and comparatively cheap price. To evaluate the pathologic mechanism or effect of a new treatment or medication, a large sized mammalian is the optimal. However, there are major limitations for their use in experimental studies. As a result, a murine animal is often chosen instead. But, in these smaller animals, there are challenges, including the relatively small size of the head and neck structures, challenges in identifying the vascular and neural anatomy and the unavailability of a surgical atlas of normal anatomy.

Previous studies suffered from two major problems. First, dissection produced trauma to the microvascular system, resulting in staining of the entire dissection field. This problem was aggravated whenever major vessels had to be removed. Second, attempts to avoid this problem, by first using formaldehyde to fix the tissues, made delicate 
dissection of the various organs difficult to perform, and identification of the arterial system, venous system and nerves difficult to accomplish. In addition, it produced artifacts and distortion between the normal anatomy and that following fixation of the tissues $(1,2)$.

Choosing the right animal model for research projects is essential, particularly when considering applicability to humans. Selecting the optimal animal species for studies in the Otorhinolaryngology Head and Neck Surgery field proves difficult, because the neck structures are complex and relatively small. Today, a variety of factors are currently considered, including the relatively short developmental period of murine animals, especially the mouse (3), the overall larger size of the rat (4), and the unique genetic or anatomical characters of the Mongolian Gerbil, Guinea Pig and Hamster. For example, the Mongolian Gerbil is generally used for research on ischemia, due to the absence of a Circle of Willis $(5,6)$, and the Hamster is used to evaluate the impact of different toothpastes on the buccal mucosa due to its large cheek pouch (7).

A detailed understanding of the specific anatomy for each murine species is important for experimental design: however, no atlas comparing the five different animals currently exists $(8,9)$. Illustrations and photo images after tissue fixation have been published, but they fail to provide a comprehensive and comparative understanding (10). In addition, in vivo, surgical documentation proves limited due to difficulties in identifying the relatively small arteries and veins without color differentiation (11). To improve visualization, researchers have used a variety of staining techniques employing Trypan blue, Toluidine blue, Alcian blue, etc. (12). But, comprehensive analysis of the entire neck region has not been completed making it impossible for researchers to use the findings for experimental modeling.

The purpose of the present study was to define the vascular anatomy in five murine species, and compare the vascular and associated anatomy among them. Vital dye staining allowed small organ identification and examination to be completed. Surgical imaging without staining was difficult to interpret due to bloodstaining of the tissues and problems with distinguishing between adjacent organs.

Based on this anatomical analysis, some animals are superior for specific studies, and should be selected for experimental design. This atlas provides researchers working in the head and neck area the information they will need to develop new, and optimal models for the future.

\section{Materials and Methods}

Experimental animals. We used five different types of adult male animals including mice (C57BL/6J, 10 weeks old, 24.7 27.5 g, n=3, DBL Co., Eumseong-gun, Chungcheongbuk-do, Korea), rats (F344, 11 weeks old, 225.5 255.5 g, SLC, Hamamatsu, Shizuoka Prefecture, Japan), mongolian gerbils (Merionesunguiculatus, 10-11 weeks old, 58.7 70.7 g, n=3, Laboratory animal resources center, Hallym university, Chuncheon-si, Gangwon-do, Korea), hamsters (Syrian, 8 weeks old, 113.1 121.3 g, n=3, SLC, Hamamatsu, Shizuoka Prefecture, Japan), and guinea pigs (Hartley, 8 weeks old, 422.1 426.7 g, n=3, SLC, Hamamatsu, Shizuoka Prefecture, Japan). The animals were housed in facilities that maintained a standard and consistent environment, with temperature: $22 \pm 2^{\circ} \mathrm{C}, 55 \pm 10 \%$ relative humidity, and a $12 \mathrm{~h}$ light and $12 \mathrm{~h}$ night Circadian cycle. Normal rodent pellet feeds (Cargill Agri Purina, Korea) and water were provided ad libitum. This animal study was performed in accordance with the guidelines of the Institutional Animal Studies Care and Use Committees of Hallym University (Hallym 2016-71).

Stain for dissection. To stain the blood vessels in each of the animals, trypan blue reagent (Cat. N. T6146, Sigma, Saintlouis, Missouri, USA) was used to perfuse each species. Animals were anesthetized with $3 \%$ isoflurane mixed oxygen (RC2+ Anesthesia System, Lab Etc. inc. Store, Clayton, Missouri, USA) and sacrificed at the end of the procedure with minimal suffering. After anesthesia, a combination of saline solution and trypan blue reagent $(0.05 \%$, mouse; and $0.1 \%$, rat, mongolian gerbil, hamster, and guinea pig) was circulated throughout the whole animal via a perfusion pump system for $5 \mathrm{~min}$. The perfusion process was terminated once the bluish stain could be seen in the auricle, nasal tip area, or liver. The tissues were fresh, not preserved, since no fixation solution $(4 \%$ paraformaldehyde) was used. As a result, the vasculature could be distinguished by the dark blue of the arterial system, and the sky blue of the venous drainage system (Figure 1) (12).

Dissection technique. After skin removal from the nose tip to the shoulder level, the overall contour of the specimen was examined. The fat tissue of the inferior neck was removed except for the submandibular and sublingual glands. At this point, the facial artery (FA) and jugular vein (JV) were identified, and the salivary glands and jugular venous system removed. The exposed sternocleidomastoid muscles were then removed and the subjacent carotid arteries under the infra-thyroid muscles were identified. Next the infra-thyroid muscles, common carotid arteries (CCA) and vagus nerves (VN) on both sides of the trachea were identified. And finally, the anterior chest wall was removed to allow identification of the aorta, as well as the vertebral arteries (VA) arising from the subclavian vessels.

Calculation of surgical images. A standardized approach was used in all species. To compare the Head and Neck surgical anatomy among the five animals, identical views were obtained, including inferior, lateral, oblique, etc. In addition, the exact location and anatomy of the JV, CCA, internal carotid artery (ICA), external carotid artery (ECA), origin of the VA were documented. To examine the more delicate structures, ophthalmic instruments (8500 Castroviejo Caliper, Fisher, Imperial, Missouri, USA) were used for dissection. To obtain the clearest visual images, the object lens of the microscope and the specimens were positioned in parallel, in order to see three dimensionally and determine exact length and area of the structures, Cannon EOS 1100D and ZEISS OPMI 1FC were used to record the images themselves. These were then analyzed using the Image $\mathbf{J}$ (https://imagej.nih.gov, USA) (Figure 1 and Table I).

Verification via cross-sectional images. To increase understanding of the anatomy for future researchers, cross-sectional morphology of all species was obtained. In addition to the vessel staining that 


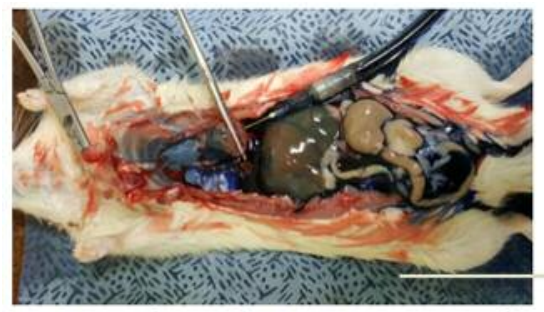

Staining of vascular system

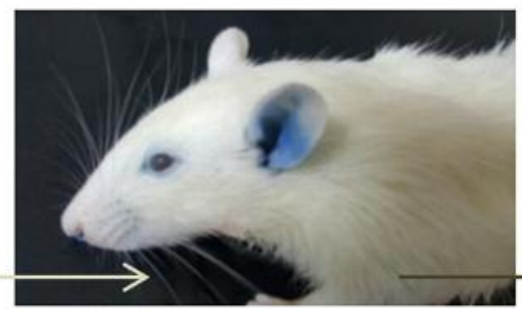

Confirmation of staining

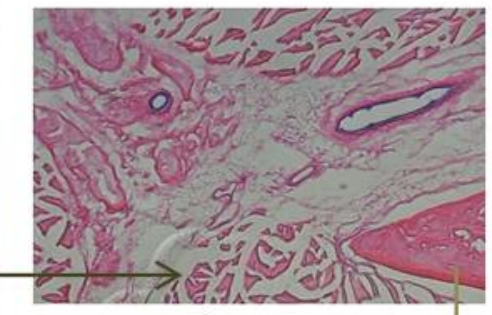

Staining verification

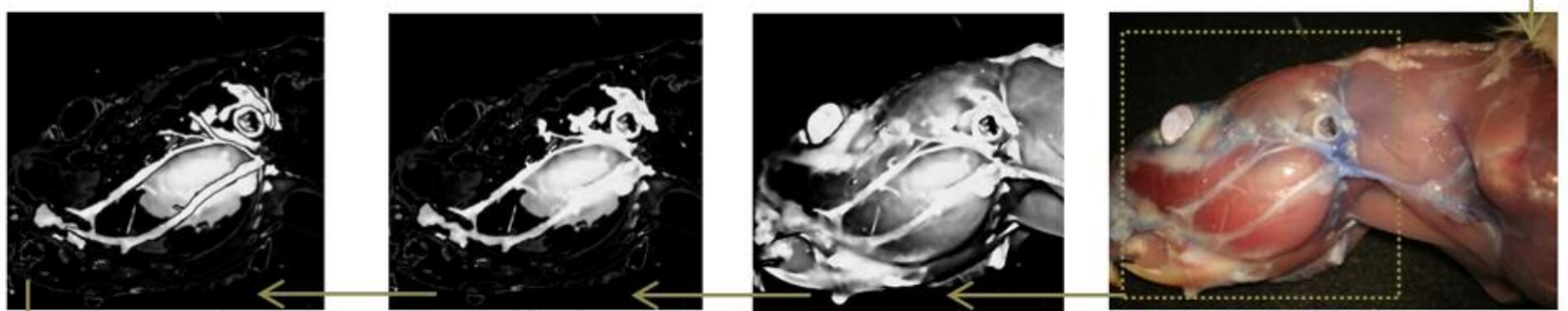

Marking for contrast Magic wand tool (//Krita.org) Image transfer, INKIFY1 (www.befunky.com)

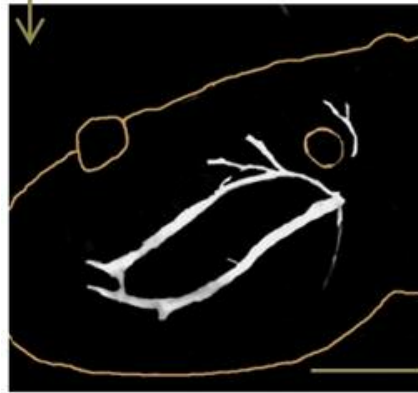

Enhancement of contrast and cartoonization

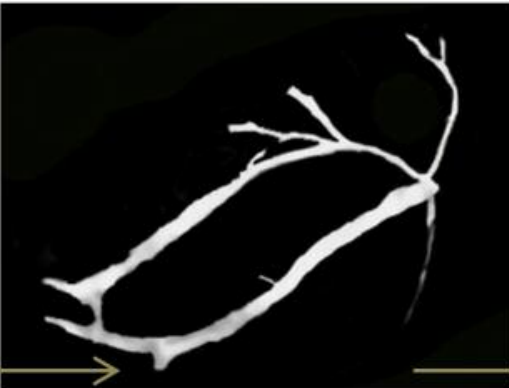

Preparation for comparing between experimental models

Figure 1. Schematic process of staining and imaging processing of the five murine head and neck organs.

had been achieved through the perfusion with the trypan blue reagent, staining was performed using an eosine $\mathrm{Y}$ reagent (cat.N.E4009, Sigma, Saintlouis, Missouri, USA) was performed for better understanding of the surrounding structures (Figure 1).

Statistical methods. For each species, a ratio of organ size to total neck size was calculated using a Kruskal-Wallis Test. The MannWhitney test was also applied post hoc test. Statistical analyses were performed using the SPSS version 21 (SPSS IBM, New York, USA). The level of significance was set at $p<0.05$.

\section{Results}

Neck anatomy of 5 murine species. Rat: The external appearance of the Rat's neck is shown in Figure 2A. The entire parotid gland (PG) was located on the facial nerve $(\mathrm{FN})$, and no deep lob of the PG was found in the present study (Figure 2B). After removal of PG, dominant zygomatic and buccal branches were identified. The temporal branch and marginal mandibular branch were relatively thin (Figure
2C). Arterial branches off of the FA were identified on both sides of the nerve supplying the surrounding tissues (Figure 2D). The JV was located on each side of the neck, on top of the sternocleidomastoid muscles (Figure 2E). The anterior and posterior branches from the face joined at the shoulder level (Figure 2J). There was no internal jugular vein (IJV). The external jugular vein (EJV) was located on the clavicle superficially, where it entered the interior of the thoracic cavity. A single belly digastic muscle was located in the inferior portion of neck, and the infrahyoid muscles were located just below it (Figure 2F). After removing the infrahyoid muscles, the CCA could be identified a moderate distance from the trachea (Figure $2 \mathrm{G}$ ) and bifurcated to two arteries (ICA and ECA, Figure 2L). Very small thyroid glands were located along the lateral portion of the larynx (Figure 2G, asterisk, dark blue stained). After removal of the anterior wall of the chest, the aorta could be seen below the thymus (Figure 2H). The facial vein was adjacent to the buccal branch of facial nerve (Figure 2I), and the brachial 
Table I. Dermographics of calculated nerves' and vessels' data.

\begin{tabular}{|c|c|c|c|c|c|}
\hline Mean (standad deviation) & Rats & Mice & Mongolian gerbils & Hamsters & Guinea pigs \\
\hline Age, weeks & $11 \mathrm{~W}$ & $10 \mathrm{~W}$ & $10-11 \mathrm{~W}$ & $8-9 \mathrm{~W}$ & $8 \mathrm{~W}$ \\
\hline Weights, mg & $225.5 \sim 255.5$ & $24.7 \sim 27.5$ & $58.7 \sim 70.7$ & $113.1 \sim 121.3$ & $422.1 \sim 426.7$ \\
\hline Marginal mandibular branch width, $\mathrm{cm}$ & $0.033 \pm .033$ & $0.047 \pm 0.047$ & $0.043 \pm 0.043$ & $0.043 \pm 0.043$ & $0.041 \pm .0410$ \\
\hline Buccal branch width, $\mathrm{cm}$ & $0.036 \pm .036$ & $0.025 \pm 0.025$ & $0.041 \pm 0.041$ & $0.045 \pm 0.045$ & $0.148 \pm 0.148$ \\
\hline Ratio of Buccal/Marginal mandibular & $1.138 \pm 0.138$ & $0.556 \pm 0.556$ & $0.964 \pm 0.964$ & $1.064 \pm 0.064$ & $3.373 \pm 0.373$ \\
\hline Sub-mandibular\&lingual area/inferior neck, $\%$ & $38.53 \pm 5.56$ & $53.53 \pm 8.66$ & $34.33 \pm 3.20$ & $64.90 \pm 13.16$ & $21.87 \pm 2.06$ \\
\hline Parotid area/lateral neck, $\%$ & $26.5 \pm 6.76$ & $23.77 \pm 2.72$ & $23.63 \pm 3.41$ & $13.18 \pm 2.99$ & $6.97 \pm 3.07$ \\
\hline Common carotid artery, Rt origin angle & $70.65 \pm 15.05$ & $54.23 \pm 32.05$ & $40.31 \pm 13.80$ & $88.77 \pm 17.77$ & $67.38 \pm 21.32$ \\
\hline Common carotid artery, Lt origin angle & $129.57 \pm 23.14$ & $110.63 \pm 4.78$ & $86.66 \pm 22.82$ & $117.89 \pm 1.57$ & $141.20 \pm 19.23$ \\
\hline Angle of carotid arteries, external and internal & $28.99 \pm 6.27$ & $39.96 \pm 8.71$ & $53.99 \pm 4.82$ & $34.22 \pm 3.51$ & $28.30 \pm 2.96$ \\
\hline
\end{tabular}

Facial neve width was calculated at mid-portion of each branch. The anterior to posterior extent of the neck was defined as from the angle of the mandible to the clavicle. The lateral neck area extended from the nuchal line to the posterior margin of the sternocleidomastoid muscle.

plexus was located at the lateral portion of the anterior scalene muscles (Figure $2 \mathrm{~K}$, asterisk). The hypoglossal nerve could be identified at the bifurcation of the CCA.

Mouse: Comparing the mouse anatomy with the rat (Figures 3A to 3L), the buccal branch of FN was located superior in relationship to the masseter muscle (Figure 3C). The supplying artery to the FN was located just below the facial branch (Figure 3D). The JV did not bifurcate in the posterior portion of neck (Figure 3F). The CCA was located just lateral to the trachea, unlike in the rat (Figure 3G). The VN was located separate from the CCA. The relative position of the hypoglossal nerve to the carotid arteries was similar (Figure 2L and Figure 3J).The deep cervical artery was located more lateral than in humans (Figure $3 \mathrm{~K}$, asterisk). The anterior and posterior arterial supplying system to the brain is shown in Figure 3L.

Gerbil: The gerbil had the biggest and thickest head and neck of the five murine species, including a very large bullae (temporal bone) bilaterally (Figure 4A-L). The PG was located more inferiorly than in the other murine species (Figure 4B). And, the vasculature appeared to be more well developed, based on the degree of staining in the present study. The blood supply to the buccal branch of the FN was located above the main FN trunk. In addition, there was more fat around the head and neck vessels than in the other murine species (Figure 4F-G).

In the gerbil, the trachea was shorter and thicker, and the thyroid was located between the trachea and esophagus (Figure 4K). After removal of the mylohyoid muscle, the hypoglossal nerve could be seen with two main branches serving to innervate the base of the tongue (Figure $4 \mathrm{H}$ ). The Initial portion of the VA was under the subclavian artery, so traction on the subclavian artery (Figure $4 \mathrm{~L}$, black arrow) was necessary to identify the VA itself (Figure 4L, white asterisk).
Hamster: The hamster (Figure 5A-L) has an extremely large cheek pouch (Figure 5A, B and I), as well as large submandibular and sublingual glands, compared to the other murine species. This makes physiological sense due to the hamster's dietary need for greater salivary secretion. In contrast, the PG was relatively small (Figure 5B). Specifically, the PG of the hamster was located in the more anterior portion of the cheek and separated from the external auditory canal. The supplying artery to the FN entered from were both side of the nerve trunk (Figure 5D). There was a single branch of the hypoglossal nerve to the base of the tongue branch (Figure $5 \mathrm{H}$ ). The anterior and posterior thyroid arteries originated from the ECA (Figure 5K). The VA originated from the subclavian artery at the level of the first rib (Figure 5L).

Guinea pig: The guinea pig (Figures 6A to 6L) has a welldeveloped platysma muscle (Figures 6A, B), and relatively, large submandibular and sublingual glands, compared to the other murine species. In contrast, there were very thin sternocleidomastoid muscles deep to the platysma (Figure 6B and $\mathrm{E}$ ). In the face, both the masseter muscle and mandible were prominent (Figures 6C and D). The branches of the ECA were easily visualized (Figure 6E and F). Three supplying arteries to the thyroid gland could be identified (Figure 6G). In the neck, both the EJV (black arrow at Figure 6I) and IJV (black arrow at Figure 6J) were present. The thyroid gland was located between the trachea and the esophagus (Figure 6K).

Comparison of size and weight of cervical structures by species: FN, vessels and glands. To compare the relative sizes of the tissues in the 5 murine species, mean values were calculated using the ALTools (http://www.altools.co.kr) (Table I). For the salivary gland determinations, the margins were obscured by the overlying fat, but could be calculated accurately following conversion to negative images. 


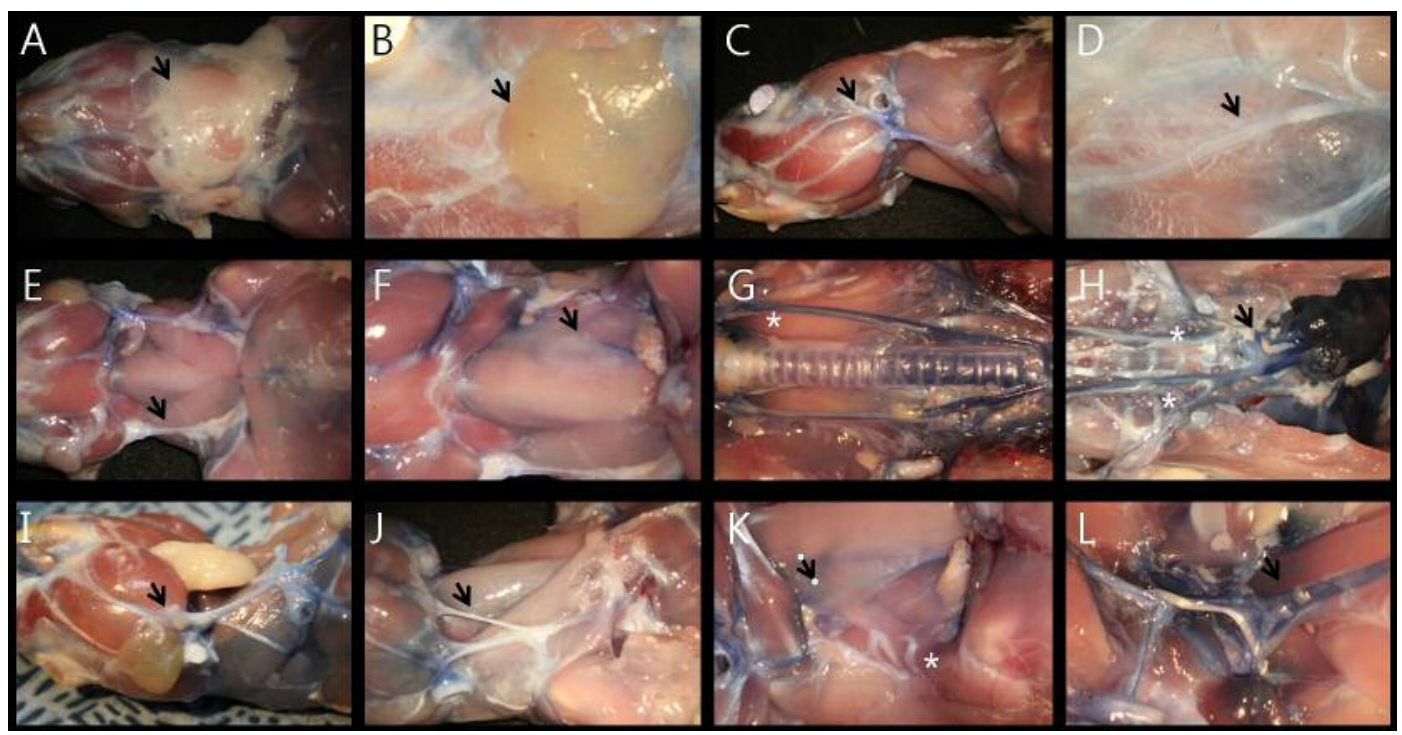

Figure 2. Vascular anatomy of rat's neck. A: After skin removal, the submandibular glands were located in the cervical fat pad (black arrow at A, front view). B: Parotid glands were located at the anterior portion of the external auditory canal (black arrow at B). C: Deep lobe of partotid gland was not present in at the rat. D: The supplying artery to the facial nerve was located on both sides of the epineurium (black arrow at D). E: Jugular vein was located on the sternocleidomastoid muscle (black arrow at E). After removal of sternocleidomastoid muscle, common carotid artery was visible on the lateral portions of the strap muscles (black arrow at F). G: Thyroid was located on the thyroid cartilage and first to third tracheal rings (white asterisk at $G$ ). H: After removal of the trachea, esophagus and thymus, aorta and both common carotid arteries were checked (black arrow at $H$ ). Vertebral arteries originated from the subclavian arteries at the level just above the first ribs (white asterisk at H). I: Facial vein was located in association with the main trunk of the facial nerve (black arrow at I). J: Two jugular veins were joined in the mid-portion of the neck on the sternocleidomastoid muscle (black arrow at J). K: After removal of sternocleidomastoid muscle, common carotid artery was visible under the infrahyoid muscles (black arrow at K). L: external and internal carotid arteries were separated at the level of thyroid cartilage and located next to the hypoglossal nerve (black arrow at $L$ ).

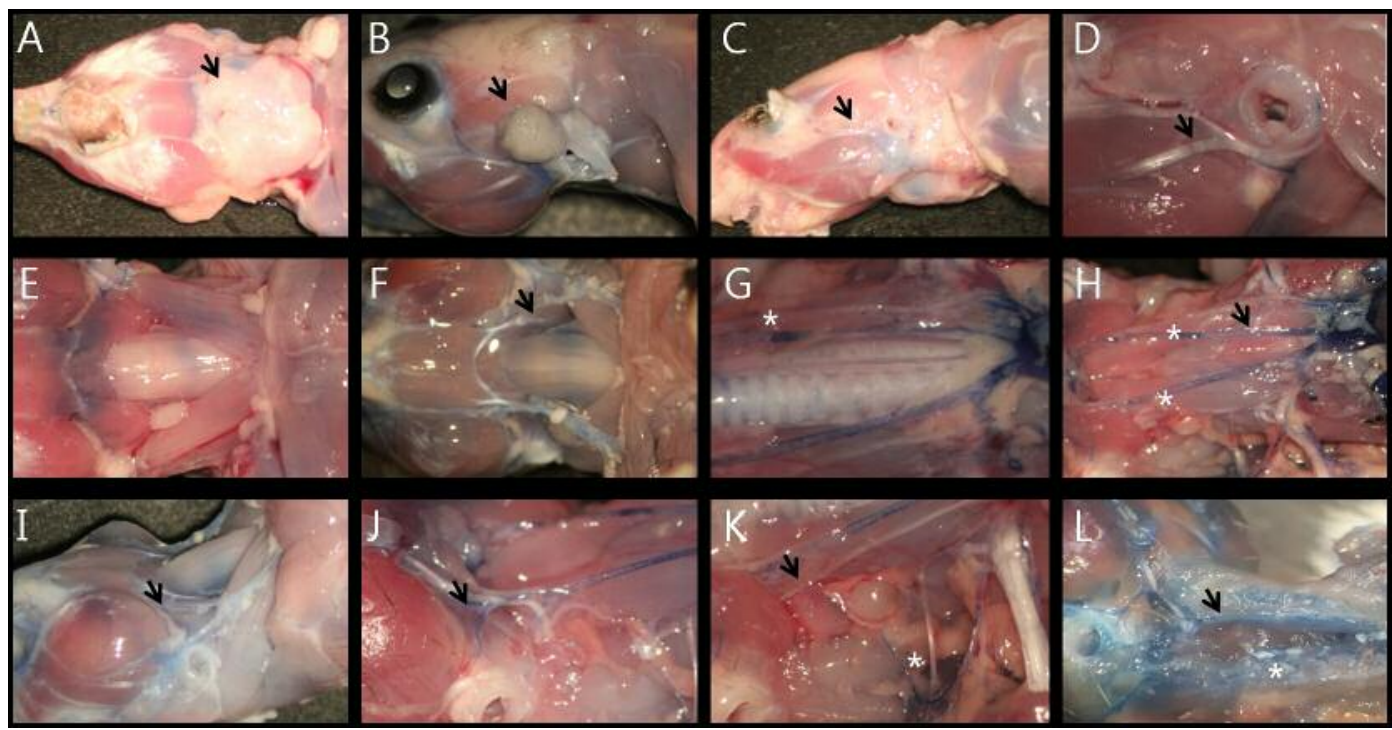

Figure 3. Vascular anatomy of the mouse's neck. A: inferior view. B: Parotid gland. C: Lateral view. D: Supplying artery to the facial nerve was located at the lower side of the epineurium (black arrow at D). E: Jugular venous system of the mouse. F: Musculature of the interior neck. G: Common carotid arteries. H: Common carotid arteries after removing trachea. I: Facial vein (black arrow at I). J: Carotid bifurcation. K: Deep cervical artery (white asterisk at K). $L$ : Anterior (internal carotid artery, black arrow at $L$ ) and posterior (vertebral artery, white asterisk at $L$ ) supplying vascular system to the brain. 


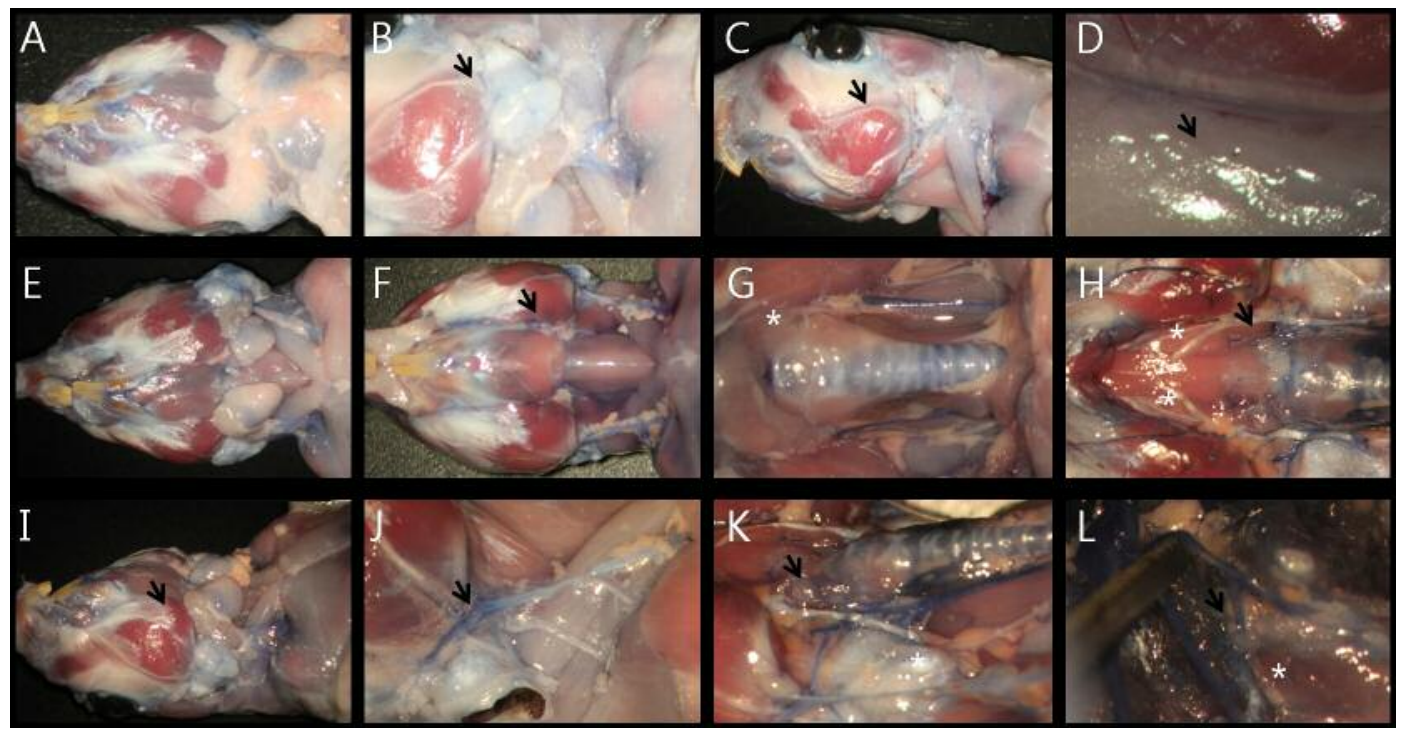

Figure 4. Vascular anatomy of gerbil's neck. A: inferior view. B: parotid gland (black arrow at B). C: lateral view, after removing the salivary glands. D: Supplying artery to the facial nerve was located at the upper edge of the epineurium (black arrow at D). E: Salivary glands. F: Muscular system and jugular veins of the interior of the neck. G: Common carotid arteries. H: Lingual arteries (black arrow at H) and bifurcated lingual nerve (white asterisk at H). I: Oblique view. J: Jugular vein, greater auricular nerve and transverse cervical nerve (black arrow at J). K: Thyroid artery (black arrow at $K$ ). L: initial portion of the vertebral artery (white asterisk at $L$ ) originating from the subclavian artery (black arrow at $L$ ).

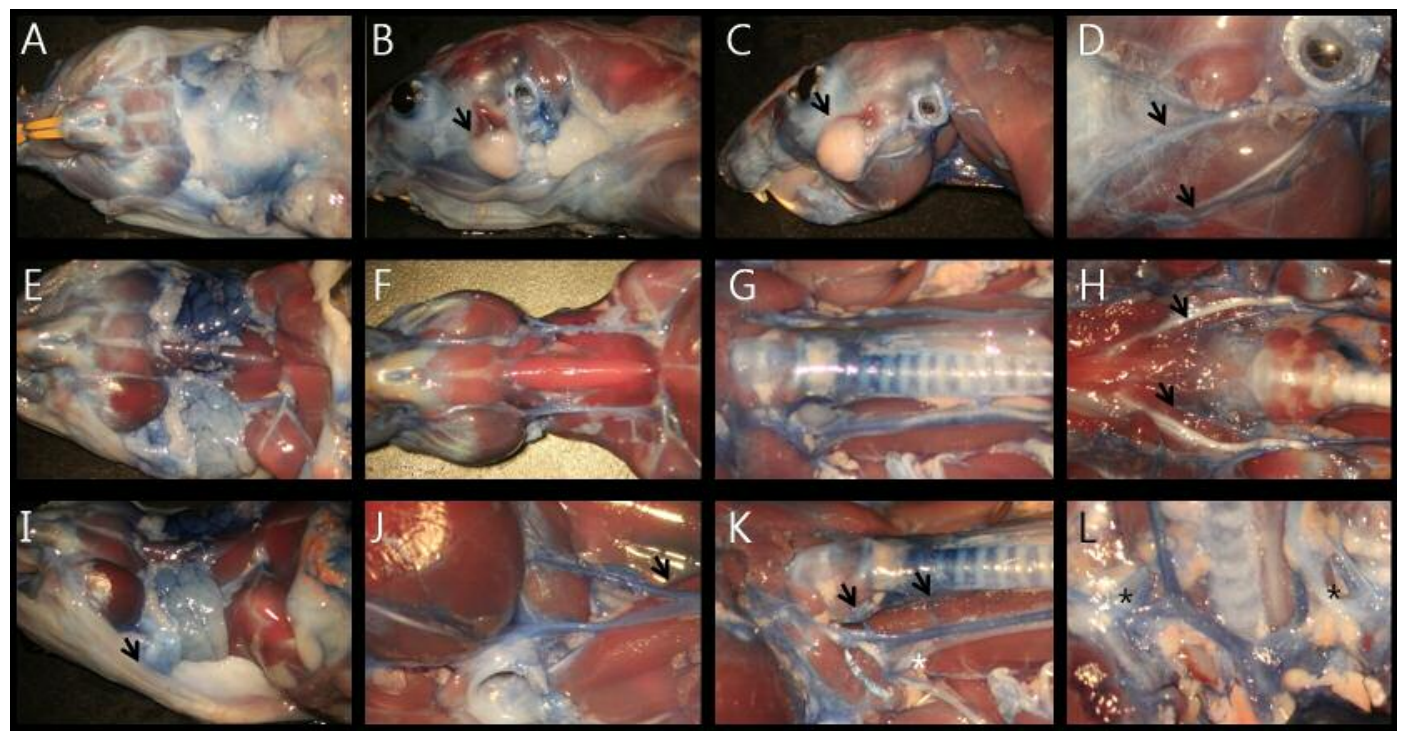

Figure 5. Vascular anatomy of the hamster's neck. A: Inferior view. B: Lateral view. C: Parotid gland (black arrow at B, C). D: Facial vessels were located adjacent to the branches of the facial nerve (black arrow at D). E: Submandibular and sublingual glands. F: Muscular system and jugular veins of the interior of the neck. G: Common carotid arteries. H: Lingual arteries and lingual nerves (black arrow at H). I: Oblique view, buccal pouch extending to the level of the first thoracic vertebra (black arrow at I). J: The jugular vein bifurcating in the middle of the neck, (black arrow at J). K: Dual supplying system of the thyroid artery (black arrow at K). L: Initial portion of the vertebral artery (black asterisk at L).

Comparison of relative organ size and calculated ratio by species. FN: the widths of the buccal and marginal mandibular branches were calculated, and compared to each other by calculating a width ratio (buccal/marginal mandibular). Each murine species had its own specific pattern of nerve branches (Figure 7A-E). In the mouse, the 


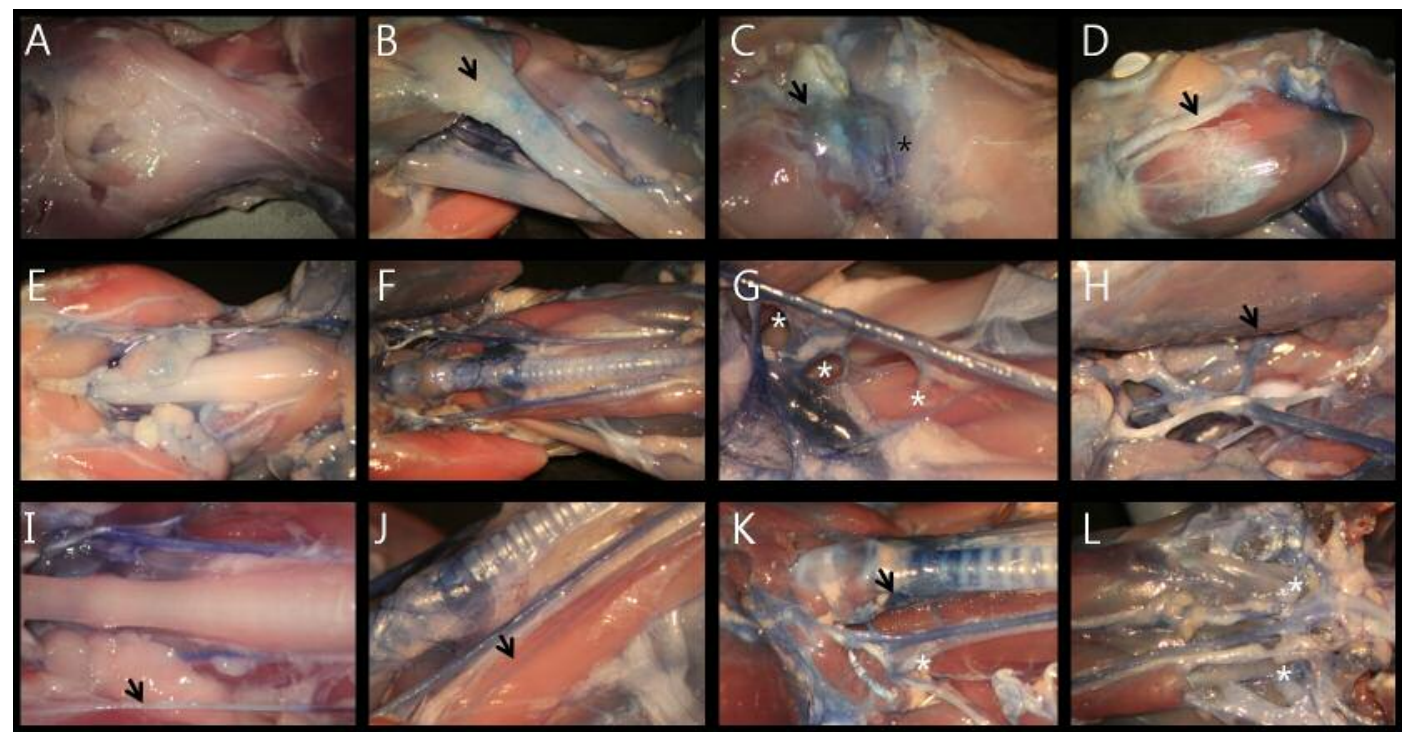

Figure 6. Vascular anatomy of the guinea pig's neck. A: Inferior view. B: Oblique view. C: Parotid gland (black arrow at C, bluish stained area). Cervical branch of the facial artery running though the parotid gland (black asterisk at C). D: Thick buccal branch of the facial nerve (black arrow at D). E: Inferior view after removal of the salivary glands and right sternocleidomastoid muscle. F: Arterial system of the interior of the neck. G: Three supplying arteries to the thyroid gland (white asterisks at G). H: Bifurcation of the common carotid artery (most anterior thyroid artery, black arrow at H). I: External jugular vein. J: Internal jugular vein. K: Thyroid gland which had been hidden behind the thyroid cartilage (dark blue area, black arrow at $K$ ). L: Initial portion of the vertebral artery (white asterisk at $L$ ).

marginal mandibular branch was thicker, and the buccal branch was thinner on a comparative basis than in the other species (Figure 7B). The mean values of the 5 murine species' nerve widths were compared (Figure 7F). The buccal branch of the Guinea Pig was relatively thicker than the other species $(p=0.021)$, In contrast there was no significant difference of the marginal mandibular branch between species. $(p=0.463)$. In addition, the buccal width/marginal mandibular width ratios were calculated and compared (Figure 7G). The greatest variance to the mean occurred in the marginal mandibular branch of the mouse and the buccal branch of the Guinea Pig $(p=0.015)$. There were no cervical branches of the $\mathrm{FN}$ in the mouse (Figure $7 \mathrm{H})$. We postulate the differences among species result from their diet and the relative degree of bruxism demonstrated by each murine species.

Salivary gland: Rather than using the conventional method of evaluation (ratio of organ to body weight), we compared the area ratio of salivary gland size to neck area. To improve accuracy, we first created negative images of the tissues (Figures $8 \mathrm{~A}-\mathrm{J}$ ). In analyzing the relative area ratios, the PG of the Hamster and Guinea pig were relatively smaller than the other species (Guinea pig was the smallest) $(p=0.008)$ (Figure $8 \mathrm{~K})$. In Contrast, the sub-mandibular and sublingual glands of the hamster were significantly larger than the other species $(p=0.012)$ (Figure 8L). The Hamster had the largest buccal pouch. Based on this study, the area of the oral cavity mucosa appeared to correlate with the size of the correlated with area of sub-mandibular and sublingual glands, but the PG did not. Since the majority of the saliva is produced by the PG, there was no direct correlation between salivary production and oral cavity mucosal size.

Arterial system: To determine the best animal model for the study of atherosclerosis and ischemia, we measured the angle of different vessels from their proximal source. To do so, in the present study, neck vascular images were extracted from the surgical photos, bifurcation angles calculated and compared to each other (Figure 9A-E, 9HL). Measurements were made between the supplying and branched artery for each of the 5 murine species (Figure 9F). The origin of the CCA from the aorta was not found to be significantly different, as a result of large standard deviation ( $p=0.192$ ) (Figure 9G), but the Hamster was noted to demonstrate an almost right angle on each side of the neck (Figure 9G, 4th graph). Based on this anatomical finding, we believe that the Hamster would serve as the best animal model for research on unilateral atherosclerosis or ischemia, particularly when a contralateral control was required. Relative to the common carotid bifurcation in the 5 murine species (Figure 9H-L). The Mongolian Gerbil had the widest angle of all of the species $(p=0.033)$ (Figure 9M). 

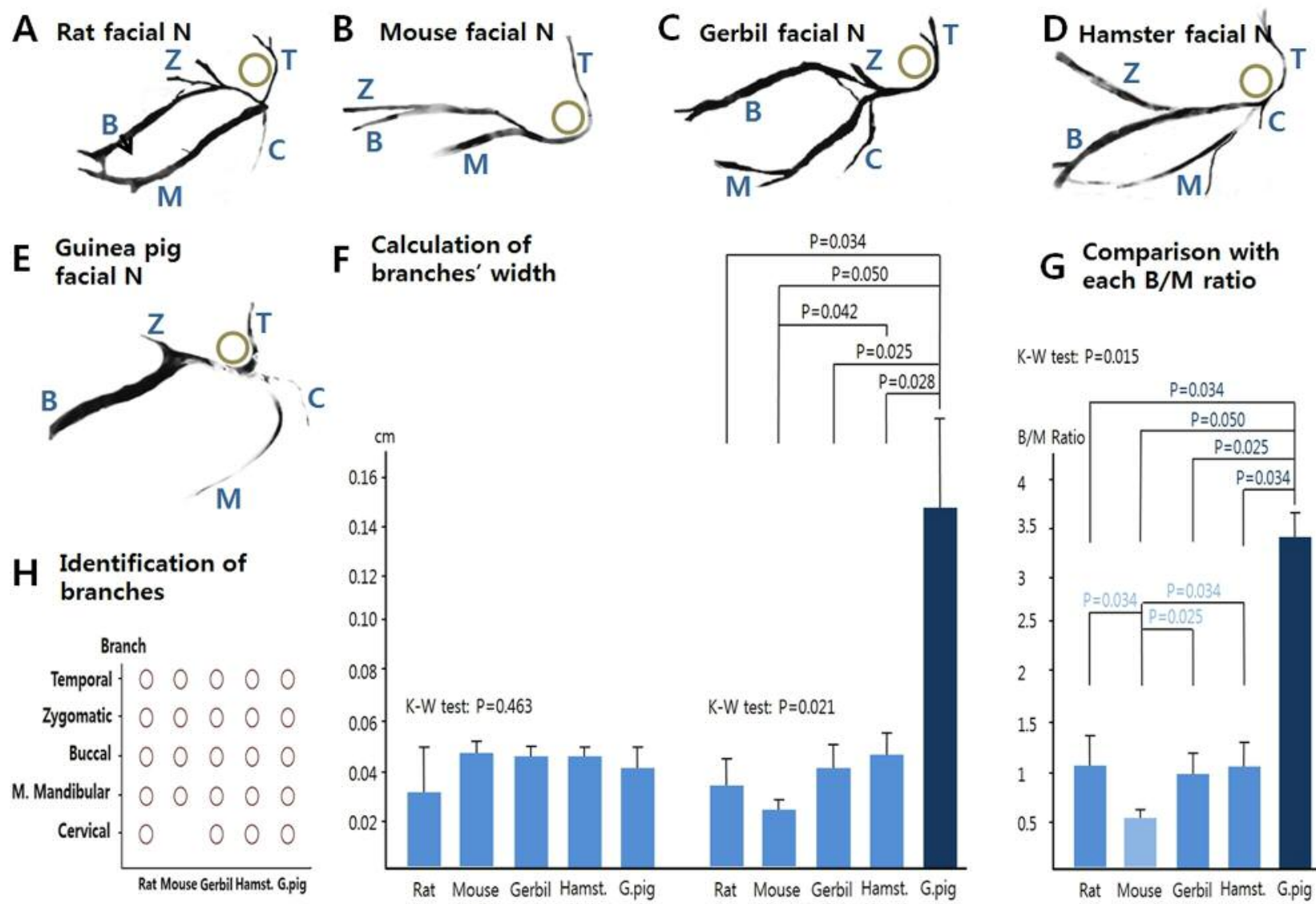

\section{G Comparison with each $\mathrm{B} / \mathrm{M}$ ratio}

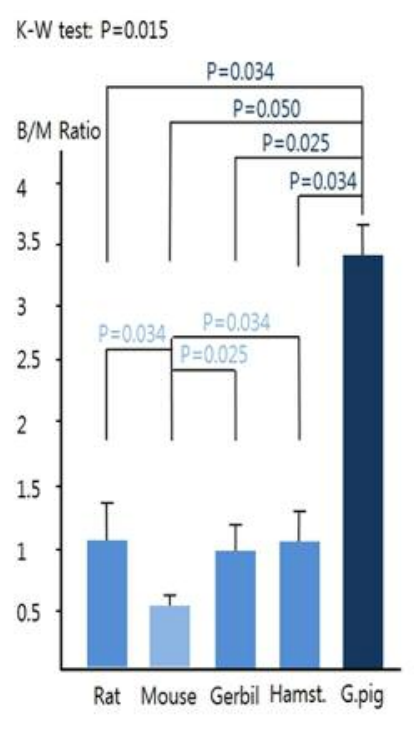

Figure 7. Comparison of width ratios of the five species' facial nerve branches, their anatomical patterns and the calculated width of each one.

\section{Discussion}

Numerous previous studies have examined the morphology of the murine head and neck structures because the murine model is an attractive surrogate for studying human biology and pathology. Previously, histological studies have been the standard approach used to define the intrinsic head and neck structures in these various murine species. To assist researchers, Jacob and Chole presented a survey atlas of normal paranasal sinus anatomy (13), while Recinos et al. reported a noninvasive means for imaging of murine cranial sutures (14). Previously, Alsmman Hassan and Abd Elhaliem Soliman reported imaging of the cornea via a paint injection method (15). Also, Lee et al. demonstrated the mouse 3-dimensional anatomy of the temporal bone (3). However, exact and clear images of the murine structures have not been published and a more comprehensive surgical atlas applicable for experimental modeling does not yet exist. To address these limitations, we have successfully imaged the head and neck vascular structures and surrounding organs of adult rodents by first perfusing the animals with trypan blue reagent. Through this approach, we avoided contaminating the dissection field with blood. And, this technique allowed near-normal touch and feeling of the instruments and tissues during the dissections. Finally, the head and neck structures remained in their normal anatomical position, throughout the experimental study itself. For otorhinolaryngology researchers in the future, the information from this study will allow better animal selection and surgical planning from what was possible in the past $(16,17)$.

Many anatomical differences between the human and murine anatomy exist. For this reason, a more accurate understanding of the comparative anatomy and more exact measurements of different parameters including organ size and relative vessel and nerve lengths allow better choices of species to be made. With accurate understanding, we might be able to predict the functional importance of organs and attached vessels systems (18).

The data from this detailed analysis provides insights for future research projects applicable to humans. The present study will be the basis of developing several new animal 
Ratio: Area of sub-mandibular $\&$ lingual glands/Area of inferior neck

A Rat

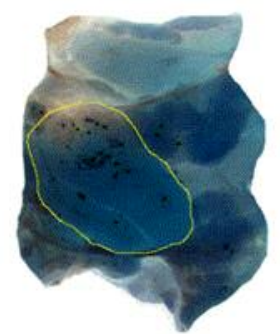

B Mouse

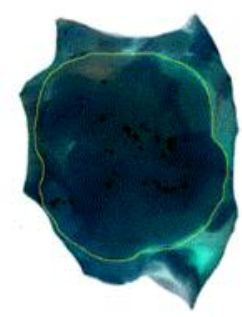

C Gerbil

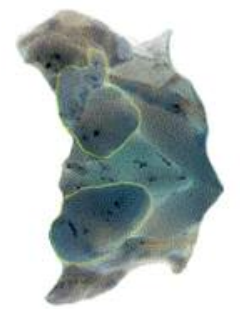

D Hamster

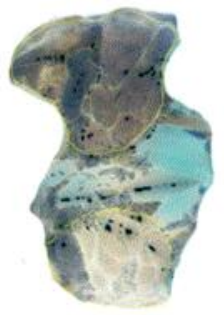

E Guinea pig

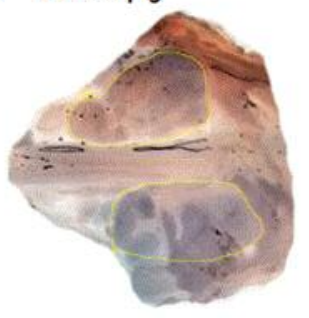

Ratio: Area of parotid glands/Area of lateral neck

F Rat

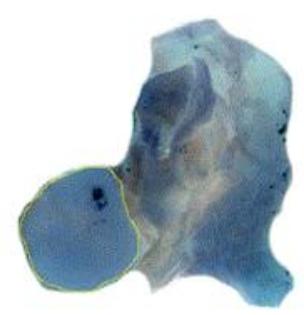

G Mouse

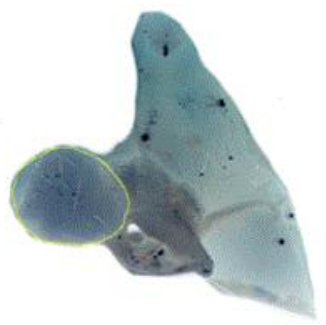

I Hamster

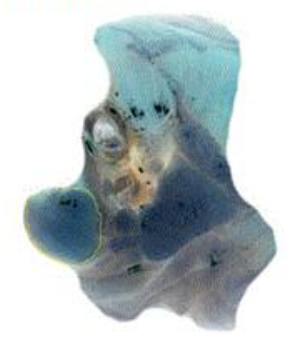

J Guinea pig

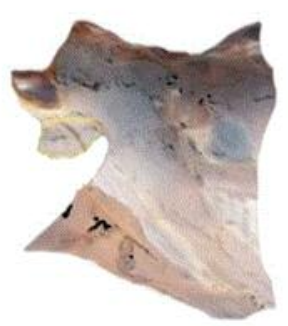

H Gerbil

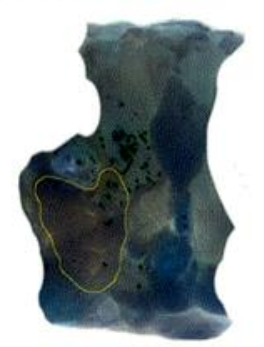

Comparison of area ratios between
five murine species

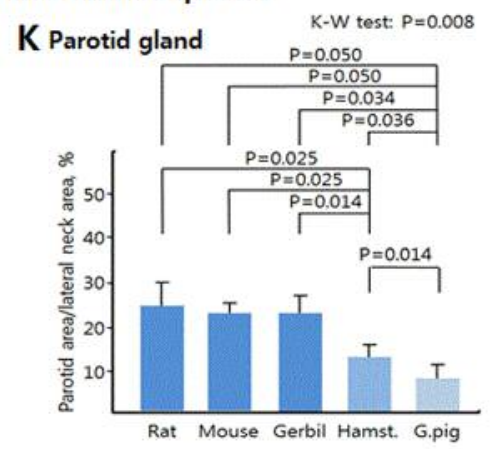

L Sub-mandibular \& Lingual glands

K-W test: $P=0.012$
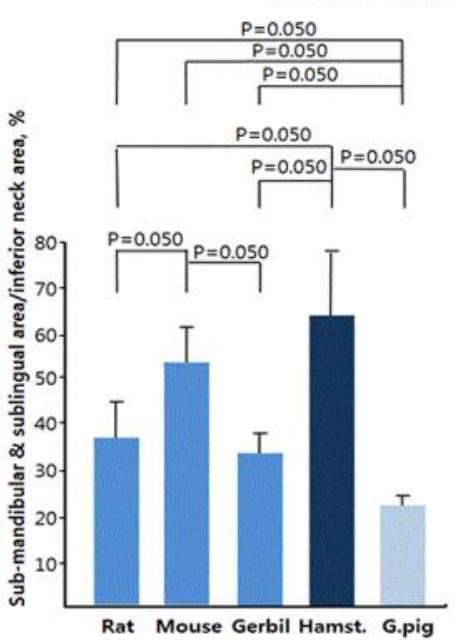

Figure 8. Comparison of width ratios of the salivary glands of the 5 species. Yellow lines in figure A-J is area calculating lines.

study models. For example, depending on the specific research design, various species would be optimal based on the variation in location of the FN branches and their relative width, the size of the different salivary glands, and the comparability of the cervical and facial vascular anatomy (19). For example, to examine the pathologic pathway and healing process following radical neck dissection including unilateral sacrification of IJV, the Guinea Pig would be the best due to its easily identifiable IJV (Figure 6J) (20).

An understanding of the exact 3-dimensional anatomy of the body is essential for research studies applicable to humans. In addition, in the laboratory, normal tissue and instrument tactile sensation is needed. The approach used in this study supports both. In the past, different approaches were used including radiological tools and digital image reconstruction to understand the three-dimensional anatomy of the head and neck $(21,22)$. However, fine tissue dissection after trypan blue perfusion, and without the use of a tissue fixative, allows for more exact analysis of the tiny structures of the murine head and neck region and retains a near normal tactile sense throughout the procedure. This combination offers many advantages, and allows the tissues, organs, blood vessels and nerves to be easily identified, preserved intact, and quantified using morphometric analysis.

The results of the present study provide future researchers with a more accurate understanding of the structural and 

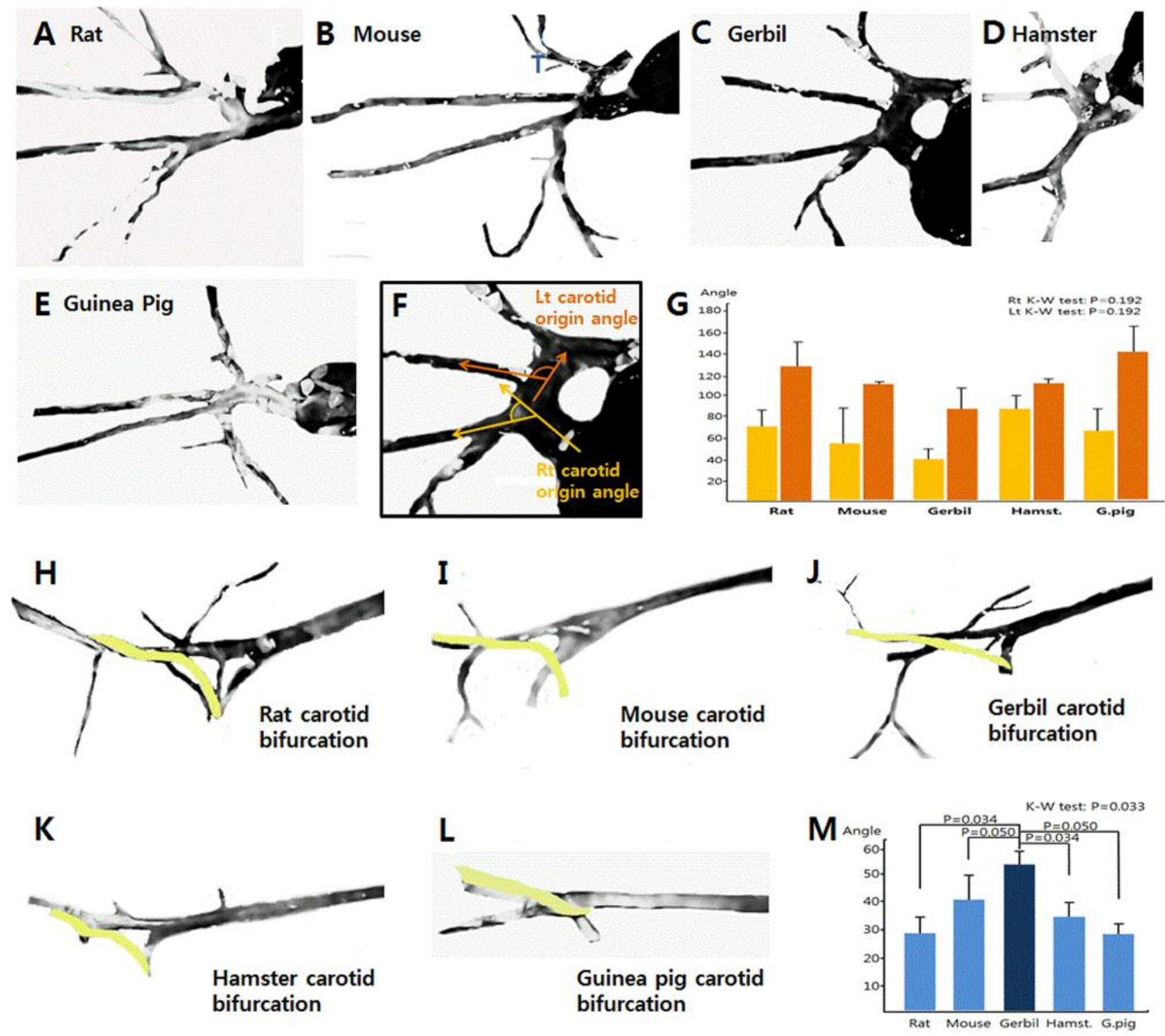

Figure 9. Comparison of the differences among the 5 species relative to the differing angles of carotid artery bifurcation.

vascular anatomy of the head and neck in different murine species. The photographs and comparative data will allow research on head and neck disease and its treatments to use a species specific anatomical basis for experimental design, and yield the highest probability for success.

In conclusion, we confirmed that a new staining method using trypan blue allows easy, accurate and undistorted identification of tissues, organs, vessels and nerves in the cervical and facial regions of different murine species. This anatomical atlas can serve as a guide for other experimental researchers as they design future projects aimed to better understand the pathology, diagnosis and treatment of different head and neck diseases.

\section{Acknowledgements}

This work was supported by Hallym University Research Fund and supported by the National Research Foundation of Korea (NRF) grant funded by the Korea government (NRF 2016R1D1A1B01014128 and NRF 2017M3A9E8033206), Republic of Korea. 


\section{References}

1 Jones ME, Werneburg I, Curtis N, Penrose R, O'Higgins P, Fagan MJ and Evans SE: The head and neck anatomy of sea turtles (Cryptodira: Chelonioidea) and skull shape in testudines. PLoS One 7: e47852, 2012.

2 Sakamoto A, Kuroda Y, Kanzaki S and Matsuo K: Dissection of the auditory bulla in postnatal mice: isolation of the middle ear bones and histological analysis. J Vis Exp 4: 119, 2017.

3 Lee JH, Park K, Kang TC and Choung YH: Three-dimensional anatomy of the temporal bone in normal mice. Anat Histol Embryol 38: 311-315, 2009.

4 Charous SJ, Hotaling JM, Burgess BD, Sappington JM, Park J, Turek $\mathrm{G}$ and Foecking EM: Muscle-nerve-muscle grafting for facial reanimation in rats. Ann Otol Rhinol Laryngol 126: 261267, 2017.

5 Kitabatake TT, Marini Lde C, Gonçalves RB, Bertolino G, de Souza HC and de Araujo JE: Behavioral effects and neural changes induced by continuous and not continuous treadmill training, post bilateral cerebral ischemia in gerbils. Behav Brain Res 291: 20-25, 2015.

6 Zhao XY, Wu CF, Yang J, Gao Y, Sun FJ, Wang DX, Wang CH and Lin BC: Effect of arginine vasopressin on the cortex edema in the ischemic stroke of Mongolian gerbils. Neuropeptides 51: 55-62, 2015.

7 Weinberg MA and Bral M: Laboratory animal models in periodontology. J Clin Periodontol 26: 335-340, 1999.

8 Lima LC, Porto ML, Campagnaro BP, Tonini CL, Nogueira BV, Pereira TM, Vasquez EC and Meyrelles SS: Mononuclear cell therapy reverts cuff-induced thrombosis in apolipoprotein Edeficient mice. Lipids Health Dis 11: 96, 2012.

9 Butz GM and Davisson RL: Long-term telemetric measurement of cardiovascular parameters in awake mice: a physiological genomics tool. Physiol Genomics 5: 89-97, 2001.

10 Cancel LM, Ebong EE, Mensah S, Hirshberg C and Tarbell JM: Endothelial glycocalyx, apoptosis and inflammation in an atherosclerotic mouse model. Atherosclerosis 252: 136-146, 2016.

11 Henstrom D, Hadlock T, Lindsay R, Knox CJ, Malo J, Vakharia KT and Heaton JT: The convergence of facial nerve branches providing whisker pad motor supply in rats: implications for facial reanimation study. Muscle Nerve 45: 692-697, 2012.
12 Kim DU, Han JW, Jung SJ, Lee SH, Cha R, Chang BS and Soh KS: Comparison of alcian blue, trypan blue, and toluidine blue for visualization of the primo vascular system floating in lymph ducts. Evid Based Complement Alternat Med 2015: 725989, 2015.

13 Jacob A and Chole RA: Survey anatomy of the paranasal sinuses in the normal mouse. Laryngoscope 116: 558-563, 2006.

14 Recinos RF, Hanger CC, Schaefer RB, Dawson CA and Gosain AK: Microfocal CT: a method for evaluating murine cranial sutures in situ. J Surg Res 116: 322-329, 2004.

15 Alsmman Hassan AH and Abd Elhaliem Soliman NG: Intrastromal injection of China Painting ink in corneas of male rabbits: clinical and histological study. J Ophthalmol 2016: 8145926, 2016.

16 Nascimento JF, Pitta GB and Mir and a Junior F: Neovascularization after surgical exposure of rat external jugular vein endothelium. Acta Cir Bras 28: 820-823, 2013.

17 Liu WW, Li CQ, Guo ZM, Li H, Zhang Q and Yang AK: Fluorescence identification of parathyroid glands by aminolevulinic acid hydrochloride in rats. Photomed Laser Surg 29: 635-638, 2011

18 Flynn JR and Bolton PS: Measurement of the vertebral canal dimensions of the neck of the rat with a comparison to the human. Anat Rec (Hoboken) 290: 893-899, 2007.

19 Aubry K, Paraf F, Monteil J, Bessede JP and Rigaud M: Characterization of a new rat model of head and neck squamous cell carcinoma. In Vivo 22: 403-408, 2008.

20 Doyle EJ, Phillips GW, Gratton MA, Long JP and Varvares MA: Minimally Invasive Approach to the Lingual and Hypoglossal Nerves in the Adult Rat. J Invest Surg 29: 144-148, 2016.

21 Beller E, Klopp D, Göttler J, Kaesmacher J, Zimmer C, Kirschke JS and Prothmann S: Closed-cell stent-assisted coiling of intracranial aneurysms: evaluation of changes in vascular geometry using digital subtraction angiography. PLoS One 11: e0153403, 2016.

22 Lin N, Ho A, Charoenvimolphan N, Frerichs KU, Day AL and $\mathrm{Du}$ R: Analysis of morphological parameters to differentiate rupture status in anterior communicating artery aneurysms. PLoS One 8: e79635, 2013.

Received June 23, 2017

Revised July 19, 2017

Accepted July 26, 2017 